\title{
Assessment of the Ovarian Reserve Before and After Laparoscopic Surgery Using Two Different Techniques for Ovarian Endometrioma
}

\author{
Hesham Abdelfattah Salem ${ }^{1}$, Hassan Masnsour Hegab ${ }^{1}$,Dalal M.Elkaffash ${ }^{2}$, \\ Hossam Azb ${ }^{1}$, Tamer A.Hosny ${ }^{1}$ \\ ${ }^{1}$ (Obstetrics and gynecology, Faculty of medicine/Alexandria, Egypt) \\ ${ }^{2}$ (Clinical pathology, Faculty of medicine/Alexandria, Egypt)
}

\begin{abstract}
The effect of two different laparoscopic methods on ovarian reserve as determined by antimullerian hormone $(A M H)$, antral follicle count $(A F C)$ and ovarian volume, in patients with ovarian endometrioma. Randomized prospective study done in tertiary education and research hospital (Shatby Alexandria university hospital) on one hundred patients presenting with ovarian endometrioma, AMH,AFC and ovarian volumes determined before and after laparoscopic surgery( postoperative first month, three months and sixth months); for two groups (Group1)cystectomy(stripping) and(Group2) bipolar coagulation performed randomly using closed envelope. The main concern was assessment of ovarian reserve damage as determined by AMH, AFC and ovarian volume, and comparing the AMH values with AMH nomogram.

The results shows that, the decreases in $A M H, A F C$ and ovarian volume were found for both coagulation and cystectomy, but the decrease was statistically significantly more frequent in cystectomized ovaries than in coagulated ovaries. Although this decrease AMH level still in normal percentile $\left(25^{\text {th }}-50^{\text {th }}\right)$ as plotted to AMH nomogram (Nelson. AMH age nomogram).
\end{abstract}

Keywords: endometrioma, laparoscopic surgery, ovarian reserve.

\section{Introduction}

Endometriosis is the presence of endometrial glands and stroma outside the uterine cavity affecting mostly women of reproductive age. The disease is usually manifested with pelvic pain, dysmenorrhea, and infertility. The prevalence is found to be $7 \%-10 \%$, but among infertile women it increases up to $50 \%$ (1). Endometrioma is the formation of a cyst within the ovary with ectopic endometrium tissue lining and is found to be in $17 \%-44 \%$ of patients with endometriosis (2). It is hypothesized that endometriomas arise as a consequence of coelomic metaplasia of the ovarian epithelium or the invagination of the inverted ovarian cortex after implantation of the endometriotic foci on the ovarian surface (3). In either case the cyst has a pseudocapsule adjacent to the normal ovarian tissue.

The treatment of endometriosis must aim at the destruction of all lesions, adhesiolysis to ensure the normal anatomical structure, prevent recurrences, and increase conception rates in patients with subfertility.

Although the most effective treatment modality of endometrioma is controversial, laparoscopic excision by the stripping technique is accepted to be the gold standard surgical approach. A meta-analysis showed that in comparison to drainage and ablative surgery, excision of endometriomas are better in terms of reduced pain, increased pregnancy, and decreased recurrence and reoperation rates(4).

One of the major concerns about excision of endometriomas is their negative effect on ovarian reserve because of follicle loss (5). Removal of endometriomas has been associated with poorer performances in IVF procedures, and decreased ovarian volumes have also been reported after surgery (6)Recently, techniques such as combining cystectomy and ablative surgery, or a three-stage procedure, have been used to reduce the damage that cystectomy causes to the ovaries $(7,8)$. Ovarian reserve has been defined as a woman's reproductive potential in terms of the number of ovarian follicles and the oocyte quality. Moreover, ovarian reserve can be screened by use of various tests (e.g., age, endocrine tests, and dynamic endocrine tests). In recent years, attention has shifted to direct tests such as AMH, ovarian volume and antral follicle count (AFC). (9-13).

To define an optimal model for the decline in circulating antimullerian hormone (AMH) with age and develop a validated age-related nomogram, Circulating AMH concentrations decline with increasing reproductive age in a manner optimally described by a quadratic equation. This validated age-related AMH nomogram will enable counseling of infertility patients regarding reproductive performance. (14)

Cystectomy may be destructive to the ovary, decreasing fertility, and ablation may be an incomplete treatment with a high risk of recurrence. Several reports comparing cystectomy with fenestration and coagulation have demonstrated that coagulation was associated with a higher recurrence rate and lower cumulative pregnancy rate when compared with cystectomy. (15-18) 
In present study our aim is to compare both techniques widely used either cystectomy or coagulation for ovarian endometrioma , and the effect on ovarian reserve(AMH,AFC and ovarian volume) and serial changes in AMH with comparing changes in AMH values with AMH nomogram.

\section{Patients And Methods}

The study included one hundred women attended the gynecological and infertility clinic of El-Shatby Maternity University Hospital presented by ovarian endometrioma between 2010 to 2012, The study Inclusion criteria was for any woman with recent ultrasonography for chocolate cyst measuring five centimeters or more unilateral or bilateral Age (18-35 years), excluding any woman with ,high basal FSH level (more than $10 \mathrm{mIu} / \mathrm{ml}$ ), Suspicious of malignancy by ultrasound, any previous ovarian surgery, any endocrinological diseases affecting ovarian reserve, and Polycystic ovary syndrome.

A total of 100 patients fulfilled the study criteria. Preoperative, postoperative first month, three months and 6 months after the surgery, the antimullerian hormone, antral follicle numbers and ovarian volumes of each patients' ovaries were assessed in the early follicular stage (days 1 to 5). The sonographic markers done using (Tellus UF-810XTD,Fukuda company, Tokyo,Japan), AFC was recorded as the total number of follicles with a diameter $<9 \mathrm{~mm}$. The ovarian volume was calculated using the prolate ellipsoid formula: Volume: $0.5233 *$ height*length*width of the ovary in $\mathrm{cm}$ ). The Preoperation ovarian volume values were obtained by removal of the cyst volume from the total ovarian volume. The AMH: preoperative and postoperative immediately, then three months and after sixth months AMH was measured by MIS/AMH ELISA DSL-1014400 (Diagnostic Systems Laboratories, Inc) Texas USA. The AMH used to compare between the two techniques to assess the effect upon ovarian reserve compare between changes in the level of AMH preoperative and at each stage during follow up periods and compare between the AMH results for both groups to the AMH nomogram.

\section{Procedure}

The hundred cases will be randomly allocated using closed envelope into two groups according to subsequent management, Laparoscopic operations were performed using video control under general anesthesia. Laparoscopic operations were performed with three $5-\mathrm{mm}$ trocars in the lower abdomen and a $10-\mathrm{mm}$ intraumbilical main trocar. We used 5-mm scissors and graspers, and normal saline solution for irrigation.

Before initiating ovarian surgery, the ovaries were completely freed with obtuse and sharp dissection. In the stripping group of ovaries, after a cleavage plane between the cyst wall and ovarian cortex was identified, the ovaries were pulled slowly and gently in opposite directions by means of two atraumatic grasping forceps. After removing the pseudocapsule from the abdominal cavity, if needed selective minimal (15 watt) bipolar coagulation of bleeding was performed, without excessive coagulation of the surgical defect to avoid damaging the ovary.

In the coagulation group of ovaries, fenestration was first performed, and a biopsy sample was obtained for histologic examination. Then the inner cyst wall was coagulated by the touch technique using $30-\mathrm{W}$ current bipolar forceps . No sutures were used in either group, and the ovaries were left open.

All operations were performed by the same surgeon. No complications occurred during or after surgery. Shifting from laparoscopy to laparotomy was not needed in any patient. Postoperatively, the pathology results were examined, and endometriosis was confirmed in all patients

\section{Statistical Analysis}

Data were fed to the computer using IBM SPSS software package version20.0.

Qualitative data were described using number and percent. Comparison between different groups regarding categorical variables was tested using Chi-square test. When more than $20 \%$ of the cells have expected count less than 5, correction for chi-square was conducted using Firsher's Exact test or Monte Carlo correction.

The distributions of quantitative variables were tested for normality using Shapiro-Wilk test and $D^{\prime}$ Agstino test, also Histogram and $Q Q$ plot were used for vision test. If it reveals normal data distribution, parametric tests was applied. If the data were abnormally distributed, non-parametric tests were used.

Quantitative data were described using mean, standard deviation, minimum and maximum. For normally distributed data, comparison between two independent population were done using independent t-test, comparison between different periods using ANOVA with repeated measures and Post Hoc test was assessed using Bonferroni adjusted. Significance test results are quoted as two-tailed probabilities. Significance of the obtained results was judged at the $5 \%$ level.

\section{Results}

The mean age of the 100 participants of the study was $27.85 \pm 2.90$ years range $20-31$ years. The mean diameter of endometriomas in the ovary cystectomy group was $6.63 \pm 0.76 \mathrm{~cm}$; that of the ovary coagulation was 
$6.64 \pm 0.74 \mathrm{~cm}$. In the coagulated ovary, the number of preoperative and postoperative follicles was compared; the precoagulation number of In the coagulated ovary the precoagulation number of AFC $9.10 \pm 1.85$ had fallen to $6.57 \pm 1.77$ after 1 month, $7.03 \pm 1.67$ after 3 months and then $7.19 \pm 1.62$ after 6 months in postcoagulation stages. In the cystectomized ovaries, the preoperative number of follicles was $9.42 \pm 2.28$, which fell to $6.11 \pm$ 2.04 after 1month, $6.23 \pm 2.09$ after 3 months and then $6.38 \pm 2.16$ after 6 months in post coagulation stages in the postcystectomy stage. The difference was statistically significant shown in (Table 1). The antral follicle numbers fell after both operations. The ovarian volume was, in the cystectomized ovaries (Group1), the preoperative ovarian volume was with mean $\pm \mathrm{SD}=11.35 \pm 2.04\left(\mathrm{~cm}^{3}\right)$, which fell to $9.25 \pm 1.56 \mathrm{~cm}^{3}$ after 1month, $7.42 \pm 1.71 \mathrm{~cm}^{3}$ after 3 months and then $7.33 \pm 1.89 \mathrm{~cm}^{3}$ after 6 months in the post cystectomy stages, In the coagulated ovary(Group2) the precoagulation ovarian volume was with mean $\pm \mathrm{SD}=11.30 \pm 2.07 \mathrm{~cm}^{3}$ had fallen to $9.61 \pm 1.81 \mathrm{~cm}^{3}$ after 1month, $8.35 \pm 1.76 \mathrm{~cm}^{3}$ after 3months and then $8.25 \pm 1.79 \mathrm{~cm}^{3}$ after 6 months in postcoagulation stages this shows significant difference as presented in(table1).

Table (1): Comparison between the two studied groups at each period according to AFC and ovarian volume parameters

\begin{tabular}{|c|c|c|c|c|c|}
\hline & Pre & & $\begin{array}{l}1^{\text {st }} \text { month } \\
\text { postoperative }\end{array}$ & $\begin{array}{l}2^{\text {nd }} \text { three months } \\
\text { postoperative }\end{array}$ & $\begin{array}{l}3^{\text {rd }} \text { sixth } \\
\text { postoperative }\end{array}$ \\
\hline \multirow{5}{*}{ 宸 } & Group I & $9.42 \pm 2.28$ & $6.11 \pm 2.04$ & $6.53 \pm 2.09$ & $6.28 \pm 2.16$ \\
\hline & Diff. & & $\downarrow 3.217^{*}$ & $\downarrow 2.652^{*}$ & $\downarrow 2.848^{*}$ \\
\hline & Group II & $9.10 \pm 1.85$ & $6.57 \pm 1.77$ & $6.73 \pm 1.67$ & $6.69 \pm 1.62$ \\
\hline & Diff. & & $\downarrow 2.529^{*}$ & $\downarrow 2.373^{*}$ & $\downarrow 2.412^{*}$ \\
\hline & $\mathbf{p}_{1}$ & 0.439 & 0.228 & 0.608 & 0.297 \\
\hline \multirow{5}{*}{ 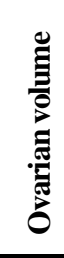 } & Group I & $11.35 \pm 2.04$ & $9.25 \pm 1.56$ & $7.33 \pm 1.89$ & $7.33 \pm 1.89$ \\
\hline & Diff. & & $\downarrow 2.093^{*}$ & $\downarrow 3.776^{*}$ & $\downarrow 3.807^{*}$ \\
\hline & Group II & $11.30 \pm 2.07$ & $9.61 \pm 1.81$ & $8.25 \pm 1.79$ & $8.25 \pm 1.79$ \\
\hline & Diff. & & $\downarrow 1.690^{*}$ & $\downarrow 2.955^{*}$ & $\downarrow 3.049^{*}$ \\
\hline & $\mathbf{p}_{1}$ & 0.911 & 0.273 & $0.009^{*}$ & $0.016^{*}$ \\
\hline
\end{tabular}

$\mathrm{p}_{1}: \mathrm{p}$ value for comparing between the two studied groups

*: Statistically significant at $\mathrm{p} \leq 0.05$

The effect on AMH values shows decrease from preoperative values Mean \pm SD $(2.98 \pm 0.75)$ in cystectomy group, and Mean $\pm \mathrm{SD}(2.79 \pm 0.71)$ in coagulation group to be during the follow up After three months postoperative the AMH values was with Mean \pm SD $(1.96 \pm 0.85)$ in cystectomy group , and Mean \pm SD $(2.46 \pm$ $0.74)$ in coagulation group and After sixth months postoperative the AMH values was with Mean \pm SD $(2.20 \pm$ $0.89)$ in cystectomy group, and Mean $\pm \mathrm{SD}(2.65 \pm 0.77)$ in coagulation group and this shows statistically significant difference between both groups as presented in table(2).

Table (2): Comparison between the two studied groups at each period according to AMH(ng/ml)

\begin{tabular}{lllll}
\hline & Pre & $\mathbf{1}^{\text {st }}$ & \multicolumn{3}{c}{ month $\mathbf{2}^{\text {nd }}$ three months $^{\text {rd }}$ sixth months } \\
\hline Group I & $6.0 \pm 2.98$ & $2.69 \pm 0.76$ & $2.16 \pm 0.85$ & $2.10 \pm 0.89$ \\
Group II & $3.80 \pm 2.79$ & $2.60 \pm 0.68$ & $2.16 \pm 0.74$ & $2.15 \pm 0.77$ \\
& & & & \\
$\mathbf{P}_{\mathbf{1}}$ & 0.194 & 0.524 & $0.002^{*}$ & $0.008^{*}$ \\
\hline
\end{tabular}

$\mathrm{p}_{1}: \mathrm{p}$ value for comparing between the two studied groups

*: Statistically significant at $\mathrm{p} \leq 0.05$

The comparison with AMH nomogram shows that according to the preoperative values we observe that in group I(cystectomy) only one patient (1.9\%) falls between $10^{\text {th }}-25^{\text {th }}$ percentiles, twenty-eight patients $(52.8 \%)$ falls between $25^{\text {th }}-50^{\text {th }}$ percentiles and twenty four patient $(45.3 \%)$ at levels $>50^{\text {th }}$ percentiles, after sixth months follow up the values of AMH when compared to the nomogram shows eight patient $(15.1 \%)$ falls between $10^{\text {th }}-$ $25^{\text {th }}$ percentiles, thirty-eight patients $(84.9 \%)$ falls between $25^{\text {th }}-50^{\text {th }}$ percentiles and no patients was $>50^{\text {th }}$ percentiles.

In group II(coagulation) the preoperative values when compared to the nomogram shows two patients (4\%) falls between $10^{\text {th }}-25^{\text {th }}$ percentiles, twenty-nine patients $(56.9 \%)$ falls between $25^{\text {th }}-50^{\text {th }}$ percentiles and 
twenty patients $(45.3 \%)$ at levels $>50^{\text {th }}$ percentiles, after sixth months follow up the values of AMH when compared to the nomogram shows six patient $(11.8 \%)$ falls between $10^{\text {th }}-25^{\text {th }}$ percentiles, thirty-nine patients $(84.3 \%)$ falls between $25^{\text {th }}-50^{\text {th }}$ percentiles and two patients $(3.9 \%)$ was $>50^{\text {th }}$ percentiles According to this data we observe although the level of AMH falls after the operative intervention it still in majority of cases between $25^{\text {th }}-50^{\text {th }}$ percentiles in both groups, this represented in table (3), figure(1).

Table (3): Comparison between AMH for age (nomogram) with pre and $3^{\text {rd }}$ stage (after 6 months) in each studied group

\begin{tabular}{|c|c|c|c|c|c|}
\hline \multirow{3}{*}{ Phase } & \multicolumn{4}{|c|}{ Group } & \multirow{3}{*}{ FEP } \\
\hline & \multicolumn{2}{|c|}{ Group 1} & \multicolumn{2}{|c|}{ Group 2} & \\
\hline & No & $\%$ & No & $\%$ & \\
\hline $\begin{aligned} \text { Pre operative } \\
\text { - } 10^{\text {th }}-25^{\text {th }} \\
\text { - } \quad 25^{\text {th }}-50^{\text {th }} \\
\text { - } \quad>50^{\text {th }} \\
\end{aligned}$ & $\begin{array}{l}2 \\
25 \\
23\end{array}$ & $\begin{array}{l}1.9 \% \\
52.8 \% \\
45.3 \%\end{array}$ & $\begin{array}{l}2 \\
29 \\
19\end{array}$ & $\begin{array}{l}4.0 \% \\
56.9 \% \\
39.2 \%\end{array}$ & 0.719 \\
\hline $\begin{array}{l}\text { Six. months } \\
\begin{array}{ll}\text { - } & 10^{\text {th }}-25^{\text {th }} \\
\text { - } & 25^{\text {th }}-50^{\text {th }} \\
\text { - } \quad & >50^{\text {th }}\end{array}\end{array}$ & $\begin{array}{l}8 \\
38 \\
0\end{array}$ & $\begin{array}{l}15.1 \% \\
84.9 \% \\
0.0 \%\end{array}$ & $\begin{array}{l}6 \\
39 \\
2\end{array}$ & $\begin{array}{l}11.8 \% \\
84.3 \% \\
3.9 \%\end{array}$ & 0.318 \\
\hline $\begin{array}{l}\text { MH statistic } \\
\mathrm{p}\end{array}$ & \multicolumn{2}{|c|}{$\begin{array}{l}56.7 \\
<0.001 *\end{array}$} & \multicolumn{2}{|c|}{$\begin{array}{l}22.8 \\
<0.001^{*}\end{array}$} & \\
\hline
\end{tabular}

FEP: $P$ value based on Fisher exact probability

MH statistic: marginal homogeneity test for related samples

$* P<0.05$ (significant

Group I: ovarian cystectomy (stripping) of endometrioma

Group II: cyst fenestration; aspiration and endo coagulation of endometrioma

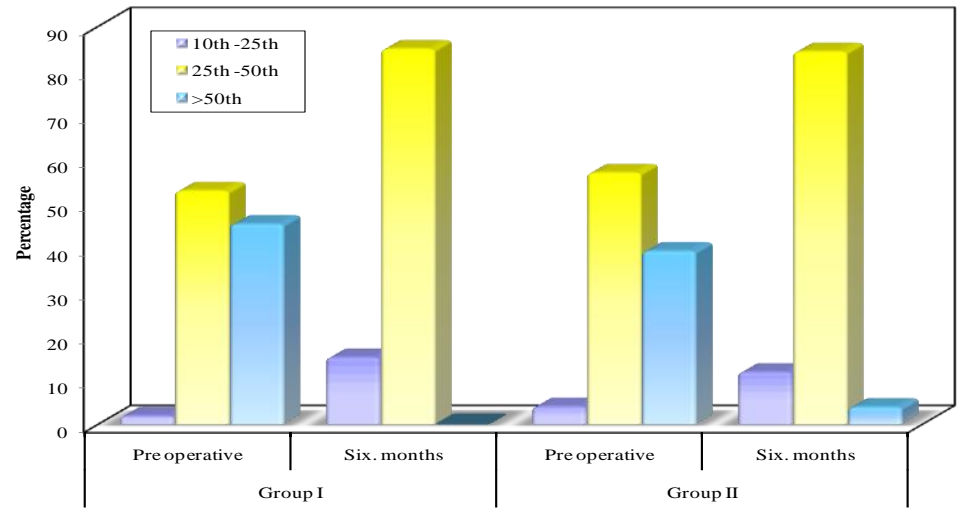

Figure (1): Comparison Between AMH For Age (Nomogram) With Pre And $3^{\text {rd }}$ Stage(After 6months) In Each Studied Group

\section{Discussion}

This study compared between two of the most frequently performed techniques for laparoscopic endometrioma surgery, we compared the laparoscopic cystectomy and laparoscopic cyst ablation using bipolar energy, mainly concerning about the effect of each technique and ovarian reserve, we observe the effect of both techniques upon the ovarian reserve when tested by various measures (AMH, FSH, AFC and ovarian volume . The best and most effective conservative laparoscopic technique for the management of endometriomas remains a controversial issue in the literature, the debate between excision and ablation exists, because the exact cause has not been found and several hypotheses have been proposed to explain the pathogenesis of ovarian endometriosis. Despite the differences between the recently proposed metaplasia theory and the implantation theory, both reached the conclusion that endometriomas are pseudocysts formed by inversion and invagination of the ovarian cortex, which contains the primordial follicles, so any surgical technique theoretically affects the ovarian tissue. ${ }^{(19,20)}$

Ovarian reserve is one of the important issues in comparing the laparoscopic techniques in removing the ovarian endometrioma, AMH one of the most reliable tests for ovarian reserve, we observe in our study 
significant reduction in AMH in both groups operated upon either by stripping or by fenestration and endocoagulation, serial follow up documents reduction early then recovers after 6 months follow up when compared to 3 months follow up but still significant reduction in relation to preoperative values. Recently a systematic review (metaanalysis) of changes in AMH after surgical excision of endometrioma shows 9 of 11 studies documented a statistically significant reduction of serum AMH level after surgery ${ }^{(21)}$. The two studies failing to document this decrease were published by the same study group (Ercan etal 2010, 2011) ${ }^{(22,23)}$

The studies evaluating serum AMH level serially after surgery documented that this reduction occurred early, being already evident at 1 week after surgery. Thereafter, levels remained stable or mildly recovered ${ }^{(24,25, \text { and } 26)}$. Against our observation,only Celik et al. ${ }^{(27)}$ observed a progressive reduction over time, but the loss of a consistent proportion of women at 6-month assessment $(26 / 65,40 \%)$ does not allow to draw definitive conclusions

To our knowledge this is the first time to compare the results with Nelson. AMH age nomogram ${ }^{(\mathbf{1 4})}$, we observed that despite the significant fall in AMH values after both techniques the values is still in normal percentiles for age mostly between $25^{\text {th }}-50^{\text {th }}$ percentiles. This validated age-related AMH nomogram will enable counseling of infertility patients regarding reproductive performance ${ }^{(14)}$, so also can be used to counsel for the effect of surgery upon the reproductive performance if this nomogram is justified. The importance of comparing the significant changes in AMH levels to validated nomogram from our point of view is to weight the risk benefit ratio when counseling patient for surgery, and subsequent effect after surgery.

Ovarian reserve also can be evaluated by sonographic assessment of AFC(antral follicle count)and ovarian volume, In our study AFC, the number of preoperative and postoperative follicles (after 1month, 3 months and 6months) was compared; In the coagulated ovary(Group2) the precoagulation number of AFC $9.10 \pm 1.85$ had fallen to $6.57 \pm 1.77$ after 1 month, $7.03 \pm 1.67$ after 3 months and then $7.19 \pm 1.62$ after 6 months in postcoagulation stages. In the cystectomized ovaries (Group1), the preoperative number of follicles was 9.42 \pm 2.28 , which fell to $6.11 \pm 2.04$ after 1 month, $6.23 \pm 2.09$ after 3 months and then $6.38 \pm 2.16$ after 6 months in post coagulation stages in the postcystectomy stage. The difference was statistically significant $\left(\mathrm{P}=0.038^{*}\right)$ and $\left(\mathrm{P}=0.037^{*}\right)$ after 3 months and 6 months comparing the two technique. Agreeing with our results, the study by Turgut Var et al, who compare the AFC in two groups, coagulated and cystectomy groups for bilateral ovarian endometrioma,the studyshows, that in the coagulated ovary, the precoagulation number of $5.42 \pm 0.77$ had fallen to $4.75 \pm 0.60$ by the postcoagulation stage. The difference was statistically significant $(\mathrm{P}=0.02)$. In the cystectomized ovaries, the preoperative number of follicles was $5.58 \pm 1.13$, which fell to $3.67 \pm 1.26$ in the postcystectomy stage. The difference was statistically significant $(\mathrm{P}=.001)$. The antral follicle numbers fell after both operations. ${ }^{(28)}$

In contrast to our study Dimitrios Tsolakidis etal, investigated the impact on ovarian reserve after laparoscopic ovarian cystectomy versus three-stage management in

patients with endometriomas as regards AFC the operated ovary increased significantly ( $\mathrm{P}=0.002)$ in group 2(three-step laser vaporization) (from 1.27-4.36) in relation to group 1 (ovarian cystectomy ) (from 2-2.38) no explanation was present for this changes. ${ }^{(13)}$

As regards ovarian volume in our study, in the cystectomized ovaries (Group1), the preoperative ovarian volume was with mean $\pm \mathrm{SD}=11.35 \pm 2.04\left(\mathrm{~cm}^{3}\right)$, which fell to $9.25 \pm 1.56 \mathrm{~cm}^{3}$ after 1month, $7.42 \pm$ $1.71 \mathrm{~cm}^{3}$ after 3 months and then $7.33 \pm 1.89 \mathrm{~cm}^{3}$ after 6months in the post cystectomy stages, In the coagulated ovary(Group2) the precoagulation ovarian volume was with mean $\pm \mathrm{SD}=11.30 \pm 2.07 \mathrm{~cm}^{3}$ had fallen to $9.61 \pm$ $1.81 \mathrm{~cm}^{3}$ after 1month, $8.35 \pm 1.76 \mathrm{~cm}^{3}$ after 3months and then $8.25 \pm 1.79 \mathrm{~cm}^{3}$ after 6 months in postcoagulation stages, our results was with agreement of Turgut Var et al, ${ }^{(28)}$ In the coagulated ovary, the ovarian volume preoperative and postoperative(after 6months) follicles was compared; the precoagulation ovarian volume $=$ $13.56 \pm 1.5$ had fallen to $9.87 \pm 2.05$ by the postcoagulation stage. The difference was statistically significant $(\mathrm{P}=0.01)$. In the cystectomized ovaries, the preoperative ovarian volume was $13.03 \pm 1.13 \mathrm{~cm}^{3}$, which fell to $6.27 \pm 1.95 \mathrm{~cm}^{3}$ in the postcystectomy stage. The difference was statistically significant $(\mathrm{P}=.001)$ both studies agree that both surgical technique significantly decrease ovarian volume with less harmful effect to the coagulation group.

In contrast to our study Dimitrios Tsolakidis etal found no effect on ovarian volume in three- stage management comparing it to stripping. ${ }^{(13)}$

Summarizing our discussion, surgery for ovarian endometrioma is debatable concern, comparing the most two technique used for laparoscopic management of endometrioma our observation was that cystectomy operation decrease the ovarian reserve significantly, but still in acceptable range as regards nomogram, the cystectomy operation is more preferable to spontaneous pregnancy rate, decrease recurrence and for more precise diagnosis for histopathological assessment excluding malignancy. 


\section{Conclusions:}

Surgery for ovarian endometrioma is debatable concern, we concluded that surgery must be tailored according to the patient complaint and presenting situation, in our study we were in concern for assessment of the effect of each type of laparoscopic surgery and ovarian reserve, concluding that ovarian reserve decreased by both technique but the decrease was statistically significantly more frequent in cystectomized ovaries than in coagulated ovaries.

We also concluded that although decrease in ovarian reserve in both technique the values of AMH levels compared to AMH nomogram (Nelson. AMH age nomogram), shows that comparing the preoperative values to sixth months postoperative the values was still in normal percentiles for age mostly between $25^{\text {th }}$ $50^{\text {th }}$ percentiles.

\section{References}

[1] ACOG practice bulletin. Medical management of endometriosis. Int J Gynaecol Obstet 2000;71:183-96.

[2] Busacca M, Vignali M. Ovarian endometriosis: from pathogenesis to surgical treatment. Curr Opin Obstet Gynecol 2003;15:321-6.

[3] Speroff L, Fritz MA. Endometriosis. In: Clinical gynecologic endocrinology and infertility. 8th ed. Philadelphia: Lippincott Williams \& Wilkins, 2011:1221-48.

[4] Vercellini P, Fedele L, Aimi G, De Giorgi O, Consonni D, Crosignani PG. Reproductive performance, pain recurrence and disease relapse after conservative surgical treatment for endometriosis: the predictive value of the current classification system. Hum Reprod 2006;21:2679-85.

[5] Exacoustos C, Zupi E, Amadio A, Szabolcs B, De Vivo B, Marconi D, et al. Laparoscopic removal of endometriomas: sonographic evaluation of residual functioning ovarian tissue. Am J Obstet Gynecol 2004;191:68-72.

[6] Garcia-Velasco JA, Mahutte NG, Corona J, Zuniqa V, Giles J, Arici A, et al. Removal of endometriomas before in vitro fertilization does not improve fertility outcomes: a matched, case-control study. Fertil Steril 2004;81: 1194-7.

[7] Donnez J, Nisolle M, Gillet N, Smets M, Bassil S, Casanas-Roux F. Large ovarian endometriomas. Hum Reprod 1996;11:641-6.

[8] Donnez J, Lousse J-C, Jadoul P, Donnez O, Squifflet J. Laparoscopic management of endometriomas using a combined technique of excisional (cystectomy) and ablative surgery. Fertil Steril 2010;94:28-32.

[9] Loverno G, Nappi L, Mei L, Giacomoantonio L, Carrierpo C, Tartagni M. Evaluation of functional ovarian reserve in 60 patients. Reprod Biomed Online2003;7:200-4.

[10] Jayaprakasan K, Al-Hasie H, Jayaprakasan R, Campbell B, Hopkisson J, Johnson I, Raine- Fenning N. The three-dimensional ultrasonographic ovarian vascularity of women developing poor ovarian response during assisted reproduction treatment and its predictive value. Fertil Steril 2009;92:1862-9.

[11] Coccia ME, Rizello F. Ovarian reserve. Ann NYAcad Sci 2008;1127:27-30.

[12] Pados G, Tsolakidis D, Assimakopoulos E, Athanatos D, Tarlatzis B. Sonographic changes after laparoscopic cystectomy compared with three-stage management in patients with ovarian endometriomas: a prospective randomised study. Hum Reprod 2010;25:672-7.

[13] Tsolakidis D, Pados G, Vavilis D, Athanatos D, Tsalikis T, Giannakou A, et al. The impact on ovarian reserve after laparoscopic ovarian cystectomy versus three-stage management in patients with endometriomas: a prospective randomized study. Fertil Steril 2010;94:71-6.

[14] Nelson SM, Messow MC, Wallace AM, Fleming R, McConnachie A. Nomogram for the decline in serum antimüllerian hormone: a population study of 9,601 infertility patients. Fertility and Sterility 2011;95(2):731-746

[15] Saleh A, Tulandi T. Reoperation after laparoscopic treatment of ovarian endometriomas by excision and fenestration. Fertil Steril 1999;72:322-4.

[16] Beretta P, Franchi M, Ghezzi F, Busacca M, Zupi E, Bolis P. Randomized clinical trial of two laparoscopic treatments of endometriomas: cystectomy versus drainage and coagulation. Fertil Steril 1998;70:1176-80.

[17] Alborzi S, Momtahan M, Parsanezhad ME, Dehbashi S, Zolghadri J, Alborzi S. A prospective, randomized study comparing laparoscopic ovarian cystectomy versus fenestration and coagulation in patients with endometriomas. Fertil Steril 2004;82:1633-7.

[18] Vercellini P, Chapron C, DeGiorgi O, Consonni D, Frontino G, Crosignani PG. Coagulation or excision of ovarian endometriomas? Am J Obstet Gynecol 2003;188:606-10.

[19] Alborzi S, Zarei A, Alborzi S, Alborzi M. Management of ovarian endometrioma.

[20] Clin Obstet Gynecol 2006;49:480-91.Gordts S, Campo R, Brosens I, Puttemans P. Endometriosis: modern surgical Management to improve fertility. Best Pract Res Clin Obstet Gynaecol 2003;17:275-87.

[21] Edgardo Somigliana, Nicola Berlanda, , Laura Benaglia,Paola Vigan_o, Paolo Vercellini, and Luigi Fedele, Surgery for endometriomas and serum AMH. Fertil Steril 2012..articla in press.

[22] Ercan CM, Sakinci M, Duru NK, Alanbay I, Karasahin KE, Baser I. Antimullerian Hormone levels after laparoscopic endometrioma stripping surgery. Gynecol Endocrinol 2010;26:468-72.

[23] Ercan CM, Duru NK, Karasahin KE, Coksuer H, Dede M, Baser I. Ultrasonographic evaluation and anti-mullerian hormone levels after laparoscopic Stripping of unilateral endometriomas. Eur J Obstet Gynecol Reprod Biol 2011;158:280-4

[24] Chang HJ, Han SH, Lee JR, Jee BC, Lee BI, Suh CS, et al. Impact of laparoscopic Cystectomy on ovarian reserve: serial changes of serum anti-M€ullerian Hormone levels. Fertil Steril 2010;94:343-9.

[25] Biacchiardi CP, Piane LD, Camanni M, Deltetto F, Delpiano EM, Marchino GL, et al. Laparoscopic stripping of endometriomas negatively affects Ovarian follicular reserve even if performed by experienced surgeons.

[26] Reprod Biomed Online 2011;23:740-6.

[27] Lee DY, Young Kim N, Jae Kim M, Yoon BK, Choi D. Effects of laparoscopic Surgery on serum anti-M€ullerian hormone levels in reproductive-aged

[28] Women with endometrioma. Gynecol Endocrinol 2011;27:733-6.

[29] Celik HG, Dogan E, Okyay E, Ulukus C, Saatli B, Uysal S, Koyuncuoglu M.

[30] Effect of laparoscopic excision of endometriomas on ovarian reserve: seriachanges in the serum antim€ullerian hormone levels. Fertil Steril 2012;97: 1472-8

[31] Turgut Var., Sertac Batioglu, ., Esra Tonguc., and Inci Kahyaoglu,. The effect of laparoscopic ovarian cystectomy versus coagulation in bilateral endometriomas on ovarian reserve as determined by antral follicle count and ovarian volume a prospective randomized study. Fertil Steril 2011;95:2247-50 